Neuropsychological disorders and higher executive functions in teens of parents with alcoholism, seen from the Roy Adaptation Model

\title{
Alteraciones neuropsicológicas y funciones ejecutivas superiores en adolescentes de padres con alcoholismo, vistos desde el modelo de adaptación de Roy
}

iD Jorge Emmanuel Mejía Benavides, iD María de Lourdes García Campos, iD Vicente Beltrán Campos

\begin{abstract}
Alcoholism by parents is closely linked to causing cognitive alterations to both the person dependent on the substance and the people with whom he lives daily, there are cognitive skills of a superior type called executive functions, which regulate the complex actions of the Being human to carry a socially accepted behavior, these functions develop in adolescence, stage in which the brain reaches its size equal to that of the adult and therefore, its maturity. Callista Roy developed a model of the cognitive process based on the functional units of the brain, which is based on the knowledge of neuroscience and observations in nursing practice, in which he mentions the executive functions and the changes suffered by the patient teen.
\end{abstract}

Keywords: executive functions, family, adolescent, Roy adaptation model.

Citación: Mejía-Benavides J. E., García-Campos M. L., Beltrán-Campos V. Alteraciones neuropsicológicas y funciones ejecutivas superiores en adolescentes de padres con alcoholismo, vistos desde el modelo de adaptación de Roy. Rev Enfer Neurol 2018;17(2):pp 43-51.

* Correspondencia: Jorge Emmanuel Recibido: 30 de octubre de 2017

Mejía-Benavides. Aceptado: 30 de marzo de 2018

Correo electrónico: je.mejiabenavides@ugto.mx

Departamento de Enfermería y Obstetricia, División de Ciencias de la Vida

Campus Irapuato-Salamanca Sede Tierra

Blanca, Universidad de Guanajuato, México

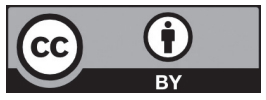




\section{Resumen}

El alcoholismo por parte de los padres está estrechamente ligado a causar alteraciones cognitivas tanto a la persona dependiente de la sustancia como a las personas con las que convive éste todos los días, existen habilidades cognitivas de tipo superior llamadas funciones ejecutivas, las cuales regulan las acciones complejas del ser humano para llevar una conducta social aceptada, dichas funciones se desarrollan en la adolescencia, etapa en la cual el cerebro alcanza su tamaño igual al del adulto y por ende, su madurez. Callista Roy desarrolló un modelo del proceso cognitivo basado en las unidades funcionales del cerebro, se fundamenta en el conocimiento de la neurociencia y las observaciones en la práctica de enfermería, hace mención de las funciones ejecutivas y los cambios que sufre el adolescente.

Palabras clave: funciones ejecutivas, familia, adolescente, modelo de adaptación de Roy.

\section{Introducción}

En la actualidad, la adicción al alcohol es un fenómeno importante en la sociedad contemporánea que conduce a graves consecuencias a nivel individual, familiar y social. ${ }^{1,2}$ Es sabido que dentro del seno familiar se generan hábitos de los cuales dependerá el desarrollo y la adaptación del adolescente al entorno en donde se desenvuelve, los hijos de consumidores de alcohol son más propensos a desarrollar alteraciones psicológicos y de comportamiento, que los coloca en alto riesgo de manifestar desórdenes durante la adolescencia tales como efectos negativos de adaptación a corto y largo plazo.

El alcohol es una sustancia psicoactiva con propiedades que causan adicción, el consumo en exceso, conlleva una pesada carga social y económica. ${ }^{3}$ El uso excesivo hace a la persona cada vez más dependiente, ocasiona trastornos de personalidad y afectaciones de conciencia, pueden presentar pesadillas, depresión con ideas o actos suicidas, desorientación, distractibilidad, alucinaciones táctiles y visuales, delirios, mal humor, paranoias, demencia y egocentrismo. ${ }^{4}$

El alcoholismo figura entre los diez principales problemas de salud pública en el mundo, por lo que su consumo en México se caracteriza por un alto índice de morbimortalidad. El alcohol tiene una larga historia de abuso y necesidad; se estima que el 30\% de la población de 18 a 65 años muestra dependencia. ${ }^{2}$ La Organización Mundial de la Salud (OMS), considera que cuando un país tiene un ingreso económico más elevado, tiende a consumir más alcohol que uno de bajo ingreso. El 16\% de los consumidores de 15 años o más, cuentan con episodios de consumo excesivo de alcohol a nivel mundial. ${ }^{5}$ El consumo de alcohol a nivel mundial durante el 2010 fue igual a 6.2 litros consumido por persona de 15 años o más, lo que se convierte en 13.5 gramos por día. Una cuarta parte del consumo corresponde al $24.8 \%$, 
adquirida de manera ilegal y el $50.1 \%$ a nivel mundial, es ingerido mediante bebidas espirituales ${ }^{5}$, es decir, aquellas bebidas con contenido alcohólico de menos del 15\% proveniente de la destilación de cereales, frutas, frutos secos y otras materias primas principalmente agrícolas. ${ }^{6}$

En América Latina, entre el 4 y $24 \%$ de la población adulta está afectada por el consumo excesivo de esta sustancia y se estima que existan en promedio 30 millones de alcohólicos. En México en el 2009, el Sistema de Vigilancia Epidemiológica de las Adiciones, reportó que el alcohol fue la droga de inicio más reportada con 49.1\% con base a información de 29417 personas de un total de 70467 que acudieron a tratamiento, de los cuales 39\% eran personas de 35 años y más, $16.8 \%$ de 15 a 19 años. En varones el 40.7\% tienen al alcohol como droga de primer impacto, mientras que las mujeres fue el $53 \%{ }^{7}$

Problemas neuropsicológicos de los adolescentes como consecuencia del consumo de alcohol en los padres

Durante la adolescencia la familia juega un papel muy importante en temas de adicciones y conductas adictivas a las cuales puede estar expuesto el individuo; sin embargo, el adolescente no tiene la confianza de platicar dichos temas con los integrantes de la familia y prefiere contarlos a sus pares o amigos, esto no siempre es la mejor elección.

El ambiente en el seno de la familia con un padre o madre con alcoholismo se caracteriza por una serie de carencias que afectan el crecimiento intelectual y emocional de los hijos tales como autoestima frágil o síntomas psicopatológicos o trastornos mentales en la edad adulta. ${ }^{9}$ Los problemas de alcoholismo pueden incrementarse al verse influenciados por los patrones de consumo de las familias. De acuerdo con la Academia de
Niños y Adolescentes de los Estados Unidos de Norteamérica, los hijos de alcohólicos tienen una probabilidad cuatro veces mayor que otros niños de convertirse en alcohólicos. Cabe mencionar que la mayoría de los hijos de alcohólicos han experimentado cierta forma de abandono o abuso. ${ }^{10}$

El buen funcionamiento familiar posibilita la adaptación a cambios propios del adolescente y se ha relacionado con la estructura de la familia; la familia de tipo nuclear sería la más preparada para afrontar los cambios en cada fase del ciclo vital. ${ }^{11}$ Los sistemas familiares funcionales crean condiciones para unas actividades parentales apropiadas y tienen su sustento en los modelos de funcionamiento de la familia ${ }^{12}$ y estas juegan un papel fundamental en la crianza de los hijos, el apoyo que les brindan los padres, contribuye a que crezcan sanos y tengan un menor riesgo de presentar problemas durante la adolescencia. ${ }^{13}$

Existe evidencia de que el consumo de alcohol puede causar alteraciones a nivel cerebral, como la pérdida de neuronas de la corteza prefrontal (CPF) en un alto grado. ${ }^{14}$ Los hijos de las personas que consumen alcohol son más propensos a desarrollar problemas psicológicos o de comportamiento, y por consecuencia están en riesgo alto de experimentar problemas durante la adolescencia, teniendo efectos negativos sobre la adaptación a corto y largo plazo. ${ }^{15}$

\section{Características psicológicas del adolescente}

La adolescencia es el periodo comprendido entre los 10 y 19 años ${ }^{16}$, biológicamente se define como la transición física marcada por el inicio de la pubertad y la terminación del crecimiento físico, cognitivo, los cambios en la capacidad de pensamiento abstracto y multidimensional o social de 
preparación para asumir el papel de adultos ${ }^{17}$, se caracteriza por una serie de cambios físicos que transforman al niño hasta la madurez física del cuerpo. $^{18}$ Durante la adolescencia, el desarrollo psicosocial normal tiene características específicas que pueden ser los determinantes sociales y culturales en los que se encuentra inmerso el adolescente, los factores biológicos como el desarrollo cerebral y las conductas propias de esta edad son un proceso voluble referente al crecimiento y desarrollo biopsicosocial de las personas. ${ }^{19} \mathrm{El}$ adolescente se puede considerar una persona vulnerable, dado los diversos cambios físicos, psicológicos y sociales de esta etapa de vida.

Los cambios que tienen los adolescentes en la forma que procesan la información se refleja en la madurez de los lóbulos frontales del cerebro, la capacidad de pensamiento abstracto conlleva implicaciones emocionales y la experiencia juega un papel importante en la determinación de cuales conexiones neuronales se atrofian y cuáles se fortalecen. ${ }^{20}$ Las operaciones formales son un nivel más alto del desarrollo cognoscitivo, donde los adolescentes perfeccionan la capacidad de pensamiento abstracto el cual aún es inmaduro ${ }^{21}$ y puede que sea competente de utilizarlo al realizar sus obligaciones, pero no al afrontar una dificultad personal. ${ }^{22}$ No obstante, muchos adolescentes se pueden adelantar al razonamiento abstracto y puede formular juicios morales complejos y realizar planes un poco más realistas para su futuro, ${ }^{20}$ teniendo la capacidad de resolver problemas para superar las dificultades. ${ }^{23}$

\section{Funciones ejecutivas superiores (FES)}

La parte del cerebro propia de las funciones cognitivas es la $\mathrm{CPF}^{24,25}$ y la principal base neuroanatómica de las funciones ejecutivas son los lóbulos frontales, mediante la cual se sustenta la funcionalidad, adaptabilidad de los procedimientos especializados que conjugan la resolución de tareas complejas. ${ }^{26}$ Los lóbulos frontales están interconectados mediante uniones corticales, subcorticales y sitios del tronco cerebral que se deben comprender como un aspecto de un sistema ejecutivo que implica estructuras del sistema nervioso central (SNC). ${ }^{27}$ El lóbulo frontal en relación con las funciones ejecutivas engloba una gran parte de la corteza cerebral y la modificación de las funciones ejecutivas se ve influenciada en el ámbito cognitivo-conductual de cada individuo, encaminadas a inferir en las habilidades para la vida y la función psicosocial. ${ }^{28}$

Las FES son un constructo donde se agrupan habilidades cognitivas implicadas en el pensamiento, atención, movimientos, supervisión, regulación, ejecución y el ajuste de conductas adecuadas para alcanzar objetivos complejos como la toma de decisiones, inhibición de respuesta y solución de problemas. ${ }^{11,14,26,27,29,30} \mathrm{La}$ capacidad principal de las funciones ejecutivas es la habilidad temporal de organizar el comportamiento y el lenguaje. ${ }^{25}$

\section{FES y sus relaciones con la crianza y el consumo de alcohol}

El consumo de alcohol influye en el SNC causando cambios en el estado de ánimo, trastornos cognitivos, aumento de la impulsividad y agresividad, lo que puede contribuir a una deficiente crianza de los hijos y un mayor riesgo de violencia, consumo de sustancias, problemas de salud mental y bajo rendimiento académico. ${ }^{31}$

El sistema familiar es el escenario donde el individuo inicia su desarrollo, por tal motivo crece rodeado de figuras de mayor influencia como sus padres, hermanos o abuelos, de quienes aprende habilidades, competencias y destrezas de 
interacción que deberán capacitarle para afrontar los diversos retos que presenta la vida ${ }^{9}$. El factor principal que interviene en el comportamiento de los adolescentes es sin lugar a duda la relación que existe entre padres e hijos; asimismo, fomentan habilidades para que desarrollen relaciones sociales positivas. ${ }^{32}$

Los sistemas familiares funcionales crean condiciones para el adecuado funcionamiento parental y tiene su sustento en los modelos de funcionamiento de la familia ${ }^{12}$, las cuales juegan un papel fundamental en la crianza de los hijos, el apoyo que les brindan los padres contribuye a que crezcan sanos y tengan un menor riesgo de tener problemas en la adolescencia. ${ }^{13}$ La percepción de crianza parental y la edad de inicio del consumo de alcohol, está relacionada positivamente, es decir; si la percepción de crianza es menor, la edad de inicio también será menor. ${ }^{33}$ Existe evidencia, sobre los hijos de alcohólicos que muestran mayor tendencia a buscar la novedad, toma de riesgos y ser más impulsivos, es decir; las funciones ejecutivas en hijos de consumidores de alcohol indican menor rendimiento a nivel cognitivo. ${ }^{34}$

\section{Resiliencia y afrontamiento del adolescente}

El interés de explicar la forma en que el ser humano afronta las experiencias adversas a lo largo de la vida ha tomado una gran fuerza en los últimos años, las influencias familiares y socio-ambientales forman parte de las experiencias adversas en la infancia (ACEs por sus siglas en inglés) dentro de las cuales se incluyen consumo de drogas por parte de los padres, pobreza, vecindario o violencia doméstica, y no sólo los sucesos dañinos del abuso emocional, físico o sexual al niño y la posibilidad de tener efectos negativos de salud en la etapa adulta. ${ }^{35,36}$ En lo referente a la resiliencia en niños, el contar con padres o cuidadores competentes es un factor que colabora en un adecuado afrontamiento a las circunstancias $^{37}$, por consiguiente, las habilidades cognitivas facultan a una persona para adaptarse y alcanzar mayores niveles de integración dentro de él mismo y con la sociedad. ${ }^{38}$

\section{Factores intrinsecos y extrínsecos que afectan las FES}

Se ha demostrado que se sabe poco acerca de cómo los niños son afectados por el consumo de alcohol por parte de los padres ${ }^{39,40}$. Un factor fundamental en el desarrollo socioemocional de los niños es la paternidad, las alteraciones en dicho desarrollo se asocian con problemas de adaptación, de un mayor riesgo de presentar trastornos del estado de ánimo y de comportamiento. ${ }^{41}$ Cuando se afecta el SNC existen cambios en todos o algunos dominios del proceso cognitivo y déficits relacionados a la habilidad de las personas ocurren dentro del dominio de alerta, atención, sensación y percepción; que son los mecanismos de entrada dentro del proceso cognitivo. ${ }^{38}$ Tirapú en su modelo del sistema cognitivo, hace mención de tres componentes básicos: entrada de información (sensorial y perceptual), sistema comparador (anticipación, selección de objetivos y elaboración de planes) y el sistema de salida (comprende la autoconciencia y la autorreflexión). ${ }^{29}$

Recomendaciones para el afrontamiento, vistas desde el modelo de adaptación de Roy

Para comprender la adaptación en los seres humanos existen diversas teorías propias de enfermería que hablan del tema, una de ellas es presentada por Sor Callista Roy, se centra en el concepto de adaptación de la persona. 
Según Roy, los sistemas humanos adaptativos son complejos y multifacéticos, reaccionan ante millones de estímulos del entorno donde se desenvuelven, mediante mecanismos de afrontamiento para adaptarse a las situaciones que se les presentan, esta capacidad de adaptación a los estímulos del entorno puede crear cambios a su alrededor para su bienestar. ${ }^{42}$ Roy afirma que el contacto con los estímulos del ambiente activa 2 subsistemas de afrontamiento, uno es el cognitivo y otro es el regulador. El cognitivo responde a través de cuatro canales cognitivo-emocionales: 1. Proceso de la información perceptiva (atención selectiva, codificación y memoria), 2. Aprendizaje, 3. Criterio y 4. Emoción. ${ }^{43}$ En este contexto, los estímulos focales del adolescente se ven relacionados desde el momento en que cuenta con un padre con adicción al alcohol, éstos actúan de manera inmediata y directa en el adolescente generándole ACEs, lo que puede conducir a desarrollarle comportamientos dañinos o negativos para la salud. Por otro lado, la etapa de vida del adolescente, tipo de relación familiar, maltrato que pueda vivir, la presión de grupo o relaciones con pares, el hecho de desenvolverse en una cultura específica, así como los recursos económicos disponibles y nivel educativo, forman parte de los estímulos contextuales, mismos que juegan un papel importante al estar evidenciados en la situación que viven los adolescentes.

Para Roy, el proceso de afrontamiento cognitivo está enmarcado en la consciencia, para los seres humanos el procesamiento de la información está detallado en tres etapas: entradas, procesos centrales y salidas, de esta forma; Roy desarrolló un modelo de los procesos cognitivos basados en tres unidades funcionales del cerebro: primera: regula la alerta, atención, sensación y percepción; segunda: la codificación, formación de conceptos, memoria y lenguaje, y tercera: regula la planificación y respuesta motora, ${ }^{43,44} \mathrm{el}$ subsistema cognitivo actúa a través de la reacción que tiene el adolescente por medio de los canales que implican el procesamiento de la información perceptiva, aprendizaje, criterio y emoción, viéndose reflejado mediante la alteración de la fluidez y flexibilidad para la ejecución efectiva de los planes de acción.

\section{Consideraciones}

Así, desde su descubrimiento realizado por Luria, los avances en el tema de las FES, han dado nuevos datos sobre el funcionamiento del cerebro, ${ }^{38}$ siendo la definición propuesta por ella, la cual en su mayoría es el término que se utiliza. De esta forma, la atención temprana a diferentes alteraciones ocurridas durante la etapa adolescente, pueden prevenir consecuencias negativas a los individuos.

\section{Conclusión}

El adolescente, como una persona que puede adaptarse a las situaciones, con capacidad de resiliencia, debe abordarse desde todas sus esferas, por tal motivo es de vital importancia tener el conocimiento de lo que ocurre con los adolescentes y su familia a nivel cerebral, dado que el personal de enfermería puede idear con base a la evidencia científica existente intervenciones específicas para la problemática que ocurre en la sociedad, y así poder contribuir con ella; actuar de manera oportuna para prevenir los daños que ocasiona en las familias, y así ser capaz de apoyar para tener una sociedad sana, responsable, capaz y con un mejor futuro para toda la población. 


\section{Agradecimientos}

A los doctores: María de Lourdes García-Campos y Vicente Beltrán-Campos por la revisión y redacción del manuscrito. Asimismo, a la Universidad de La Sabana, Chía Cundinamarca, Colombia y Campus Celaya-Salvatierra, Universidad de Guanajuato, México donde se realizó esta investigación.

\section{Referencias}

1. Ciubară A, Burlea SL, Săcuiu I, Radu D, Untu I, Chiriță R. Alcohol addiction-a psychosocial perspective. Procedia Soc Behav Sci [Internet]. 2015;187:536-40. Disponible en: doi. org/10.1016/j.sbspro.2015.03.100

2. Rojas E, Real T, García-Silberman S, Medina-Mora ME. Revisión sistemática sobre tratamiento de adicciones en México. Sal Ment [Internet]. 2011;34(4):351-65. Disponible en: https://bit.ly/2H500vh

3. Organización Mundial de la Salud. Alcohol. [Internet]. [Acceso 10 septiembre 2017]. Disponible en: https://bit.ly/2rLoVw2

4. Escalona J, Leyva D, Benítez T, Vázquez $\mathbf{O}$. Las funciones ejecutivas en pacientes alcohólicos. Psicol Am Lat [Internet]. 2011;(21):14-48.

5. Organización Mundial de la Salud. Global status report on alcohol and health. [Internet]. [Acceso 10 septiembre 2017]. Disponible en: https://bit. ly/2C7i9Fd

6. Diario Oficial de la Unión Europea. Reglamento (CE) No 110/2008 del Parlamento Europeo y del Consejo. [Internet]. [Acceso 12 septiembre 2018]. Disponible en: https://bit.ly/2m1PooX

7. Instituto Nacional de Psiquiatría Ramón de la Fuente Muñiz. Encuesta Nacional de Adicciones 2011: reporte de alcohol. [Internet]. [Acceso 13 febrero 2016]. Disponible en: https://bit. ly/1yA6bxB

8. Pérez A, Pérez R, Martínez ML, Leal FJ, Mesa I, Jiménez I. Estructura y funcionalidad de la familia durante la adolescencia: relación con el apoyo social, el consumo de tóxicos y el malestar psíquico. Aten Prim [Internet]. 2007;39(2):61-7. Disponible en: https://bit.ly/2VLYuGw

9. Jiménez $\mathbf{M}$, Serra J, Villafañe $\mathbf{A}$, Jiménez $\mathbf{W}$. Hijos adultos de madres/padres alcohólicos y factores de riesgo psicológicos en estudiantes universitarios. Revista Electrónica "Actualidades Investigativas en Educación”. [Internet]. 2011;11(1):120. Disponible en: https://www.redalyc.org/ articulo.oa?id $=44718060007$

10. American Academy of Child and Adolescent Psychiatry. Los hijos de alcohólicos. [Internet]. [Acceso 14 Julio 2015]. Disponible en: https:// bit.ly/2PLHOEQ

11. Merchán-Naranjo J, Boada L, del Rey-Mejías A, Mayoral M, Llorente C, Arango C, et al., La función ejecutiva está alterada en los trastornos del espectro autista, pero esta no correlaciona con la inteligencia. Rev Psiquiatr Salud Ment [Internet]. 2016;9(1):39-50. Disponible en: doi. org/10.1016/j.rpsm.2015.10.005

12. Matejevic M, Jovanovic D, Lazarevic V. Functionality of family relationships and parenting style in families of adolescents with substance abuse problems. Procedia Soc Behav Sci [Internet]. 2014;128:281-7. Disponible en: doi. org/10.1016/j.sbspro.2014.03.157

13. Kumpfer KL, Johnson JL. Intervenciones de fortalecimiento familiar para la prevención del consumo de sustancias en hijos de padres adictos. Adicciones [Internet]. 2007;19(1):13-25. Disponible en: https://bit.ly/2DTO2mf

14. Villegas-Pantoja MA, Alonso-Castillo MM, Benavides-Torres RA, Guzmán-Facundo FR. Consumo de alcohol y funciones ejecutivas en adolescentes: una revisión sistemática. Aquichan [Internet].2013;13(2):234-46. Disponible en: h t t p : / / w w w.redalyc.org / articulo. oa?id $=74128688015$

15. Finan LJ, Schulz J, Gordon MS, Ohannessian CM. Parental problem drinking and adolescent externalizing behaviors: The mediating role of family functioning. $J$ Adolesc [Internet]. 2015;43:100-10. Disponible en: doi. org/10.1016/j.adolescence.2015.05.001 
16. Organización Mundial de la Salud. Desarrollo en la adolescencia. [Internet]. [Acceso 14 septiembre 2017]. Disponible en: https://bit.ly/1WldmmP

17. Martínez MR, Ramírez L. La salud del niño y del adolescente: crecimiento y desarrollo. Manual Moderno, México, 2009.

18. Abadía GI. Características del desarrollo en el adolescente. En: Araújo VAM, García QMC, Sierra LJA, Abadía GI, Gálvez SAM, Arteaga DJ. Adolescencia. Chía, Colombia: Universidad de La Sabana; 2000.

19. Gaete V. Desarrollo psicosocial del adolescente. Rev Chil Pediatr 2015;86(6):436-43.

20. Papalia DE, Sally WO, Ruth DF, Ortiz SMH, Hernández LJS, López CMA. Desarrollo bumano. McGraw Hill. Colombia, 2012.

21. Opladen T, Cortes-Saladelafont E, Mastrangelo M, Horvath G, Pons R, Lopez-Laso E, et al., The International Working Group on Neurotransmitter related disorders (iNTD): A worldwide research project focused on primary and secondary neurotransmitter disorders. Mol Genet Metab Rep [Internet]. 2016;9:61-66. Disponible en: doi.org/10.1016/j.ymgmr.2016.09.006

22. Cynthia Holland-Hall C, Burstein GR. Desarrollo en la adolescencia. En: Kliegman RM, Stanton BF, St Geme JW, Schor NF, Behrman RE. Tratado de pediatría. Madrid, España: Elservier; 2016.

23. Katzman DK, Neinstein LS. Medicina de la adolescencia. En: Goldman L, Schafer AI. Tratado de medicina interna. Madrid, España: Elservier; 2017.

24. Goto Y, Yang CR, Otani S. Plasticidad sináptica funcional y disfuncional en la corteza prefrontal: papel en los trastornos psiquiátricos. Psiquiatr Biol [Internet]. 2011;18(1):18-27. Disponible en: doi. org/10.1016/j.psiq.2009.08.001

25. Delgado-Mejía I, Etchepareborda M. Trastornos de las funciones ejecutivas. Diagnóstico y tratamiento. Rev Neurol [Internet]. 2013;57(1):95-103. Disponible en: https://bit. ly/2DRcq8g

26. Verdejo-García A, Bechara A. Neuropsicología de las funciones ejecutivas. Psicothema. 2010; 22(2):227-35.
27. Yuan P, Raz N. Prefrontal cortex and executive functions in healthy adults: a meta-analysis of structural neuroimaging studies. Neurosci Biobehav R [Internet]. 2014;42:180-92. Disponible en: doi. org/10.1016/j.neubiorev.2014.02.005

28. Andrade J, Rodríguez A, Campos J. GABA, depresión y suicidio: aspectos epigenéticos asociados. Psicologia Com. 2014;18.

29. Alvis A, Arana C, Restrepo J. Propuesta de rehabilitación neuropsicológica de la atención, las funciones ejecutivas y empatía en personas con diagnóstico de trastorno antisocial de la personalidad, desvinculadas del conflicto armado colombiano Rev Virtual Universidad Católica del Norte 2014;2(42):138-53.

30. Peeters M, Janssen T, Monshouwer K, Boendermaker W, Pronk T, Wiers R, et al., Weaknesses in executive functioning predict the initiating of adolescents' alcohol use. Develop Cog Neurosc [Internet]. 2015;16:139-46. Disponible en: doi.org/10.1016/j.dcn.2015.04.003

31. Lund I, Bukten A, Storvoll E, Moan I, Skurtveit S, Handal M, et al., A cohort study on long-term adverse effects of parental drinking: background and study design. Substan abuse: Res Treat [Internet]. 2015;9(2):77-83. Disponible en: doi.org/10.4137/SART.S23329

32. Martínez B, Musitu G, Amador L, Monreal M. Estatus sociométrico y violencia escolar en adolescentes: implicaciones de la autoestima, la familia y la escuela. Rev Lat Psic 2012;44(2):55-66.

33. Lugo L, Arteaga D, Aguilar H. Funciones ejecutivas superiores y su relación con características psicométricas en adolescentes. Jóvenes Ciencia 2016;2(1):1515-9.

34. Acosta MR, Juárez F, Cuartas M. Funciones ejecutivas y antecedentes familiares de alcoholismo en adolescentes. Pensamiento Psicol [Internet]. 2018;16(1):57-68. Disponible en: doi. org/10.11144/Javerianacali.PPSI16-1.feaf

35. Balistreri K, Alvira-Hammond M. Adverse childhood experiences, family functioning and adolescent health and emotional well-being. Public Health [Internet]. 2016;132:72-8. Disponible en: doi.org/10.1016/j.puhe.2015.10.034 
36. Anda RF, Whitfield CL, Felitti VJ, Chapman D, Edwards VJ, Dube SR, et al., Adverse childhood experiences, alcoholic parents, and later risk of alcoholism and depression. Psychiat Serv [Internet]. 2002;53(8):1001-9. Disponible en: doi.org/10.1176/appi.ps.53.8.1001

37. Vera B, Carbelo B, Vecina M. La experiencia traumática desde la psicología positiva: Resiliencia y crecimiento postraumático. Papeles Psicólogo 2006; 27(1):40-9.

38. Roy C. Alterations in cognitive processing. En: Stewart-Amidei C, Kunkel JA. AANN's Neuroscience nursing: buman responses to neurologic dysfunction. Pennsylvania: Saunders; 2001.

39. Rossow I, Felix L, Keating P, McCambridge J. Parental drinking and adverse outcomes in children: a scoping review of cohort studies. Drug Alcohol Rev [Internet]. 2016;35:397-405. Disponible en: doi.org/10.1111/dar.12319

40. Rossow I, Keating P, Felix L, McCambridge J. Does parental drinking influence children's drinking? A systematic review of prospective co- hort studies. Addiction [Internet]. 2016;111(2):204217. Disponible en: doi.org/10.1111/add.13097

41. Lomanowska AM, Boivin M, Hertzman C, Fleming AS. Parenting begets parenting: a neurobiological perspective on early adversity and the transmission of parenting styles across generations. Neurosc [Internet]. 2017;342:12039. Disponible en: doi.org/10.1016/j. neuroscience.2015.09.029

42. Phillips KD, Harris R. Modelo de adaptación. En: Alligood M, Marriner-Tomey A. Modelos y teorias en enfermería. Madrid, España. Elservier, 2015.

43. Roy C. The Roy adaptation model. New Jersey, Pearson, 2009.

44. Gutiérrez C, Veloza M, Moreno M, Durán M, López C, Crespo O. Validez y confiabilidad de la versión en español del instrumento "escala de medición del proceso de afrontamiento y adaptación" de Callista Roy. Aquichan 2007; $7(1): 54-63$. 